\title{
WORD SENSE DISAMBIGUATION: A REVIEW
}

\author{
School of Computer Science \\ Faculty of Information Science \& Technology \\ Albydha University, Rada'a, Albydha, Yemen \\ Qasemacc22@gmail.com
}

\section{INTRODUCTION}

In the process of natural language, a lot of words have different connotations. The correct sense of a word depends upon the context in which the word occurs. Word sense disambiguation known as the process of selecting the most correct sense of the word in a given sentence. Furthermore, Most of natural language processing applications, such as the extraction of information, machine translation, and Analysing of content are supported by the process of word sense disambiguation which can be an essential pre-processing step for them. Figure 1 shows the general process of word sense disambiguation.

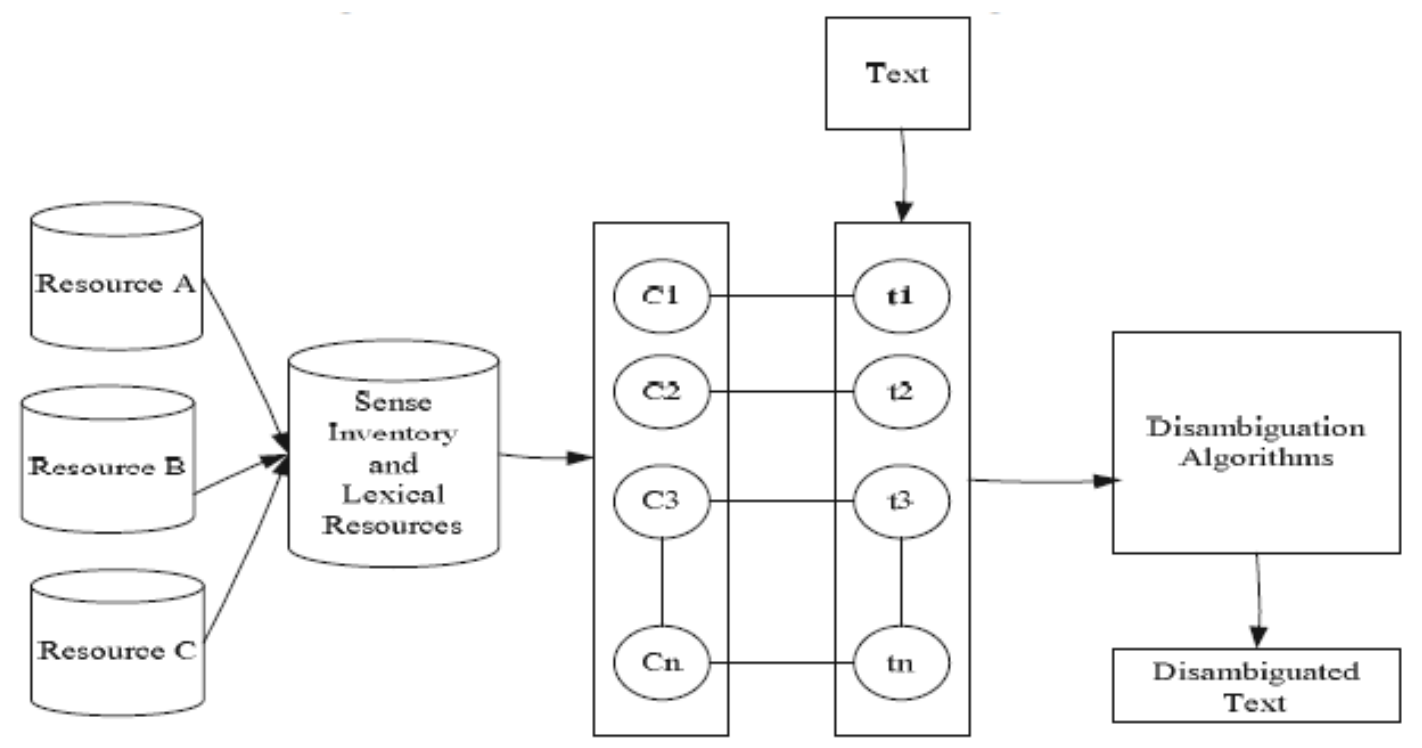

Figure 1: General model of word sense disambiguation

Furthermore, in English language words can have fully opposite meanings in different contexts. An example to show that, the sentence "The bank of Yemen was robbed", bank here means the institution of finance. On the other hand, in the "My home town is on the southern bank of Yemen" the word bank here means a shore of a river or a land.

There are many approaches for word sense disambiguation one of them is knowledge based word sense disambiguation which in our case assign the senses to each word "bank" and 
indicates which sense is correct by word overlapping between the word in the given sentence and the word from WordNet, then the correct sense is the one which have the maximum number of overlapping, in our case we have the sense "finance" which can be in the following words [finance, deposit, money, activity, accept], and the following words for the sense "land" [river, water, body, land] the total of overlapping will be:

- First Sentence: (land $=0$, finance $=2$ )

- Second Sentence: $($ land $=1$, finance $=0)$

Therefore, the finance sense is assigned to the first sentence and the land sense assigned to the second sentence. However, large number of suitable sense in WordNet is undistinguishable from each other in this method. Whereas the supervised methods are obtained to be more suitable than other approaches such as unsupervised method and knowledge base approaches.

\section{CALLENGES IN WORD SENSE DISAMBIGUATION}

\section{Differences between dictionaries}

In some cases, many senses are closely related to each other so it's difficult to the separate the words into senses.

\section{Part-of-speech tagging}

Sense tagging and Part of speech tagging are closely correlated to each other. So the decision of whether these tasks should be decoupled or kept together becomes much more difficult.

\section{Human Interaction}

Human interaction provides the better result than computer to find the sense of words. But it is difficult for human to memorize all the possible senses of words.

\section{Common sense}

Sometimes it is difficult to parse the meaning of words without common sense. For example, "Ram and Shyam are fathers" and "Ram and Shyam are brothers". In first sentence each is independently a father whereas in second sentence they are brothers of each other.

\section{Sense Inventory and algorithms task-dependency}


It is difficult to find the accurate sense of word between multiple languages. An example, the ambiguity of word "mouse" where is it animal or computer device is not pertinent in ArabicEnglish machine translation.

\section{RELATED WORK}

1. A Research paper was proposed by Pradeep Sachdeva 2014, “An Improved Approach to Word Sense Disambiguation" [1].

The problem: Improvement of the Previous Algorithms which use only two factors to found the similarity between the nearby and target words, first factor calculating the intersection between the glosses of the nearby and target words. Second factor by identifying the exact occurrence of the nearby word's sense in the hierarchy (hypernyms) of the target word's senses.

\section{Solution:}

Providing a Knowledge based algorithm for disambiguating words using the tool of computational linguistics. This approach includes more factors for calculating similarity which are the whole hierarchy, intersection, and distance which has not been involved by any of the previous approaches. Choosing the correct sense based on the highest score.

Data set: Only noun words polysemous content of SemCor dataset.

Result: The proposed Knowledge based approach as shown in table 1 resulted with a higher precision for the three top result measurement which is better than other approaches.

Table 1: results of knowledge based approach with different approaches tested for nouns.

\begin{tabular}{|c|c|c|c|c|}
\hline \multicolumn{2}{|c|}{ Measure } & Precision & Recall & F1 Measure \\
\hline & $\mathbf{5 3 . 3}$ & $\mathbf{4 3 . 6}$ & $\mathbf{4 7 . 9}$ \\
\hline $\begin{array}{c}\text { Graph Connectivity } \\
\text { Measure[7] }\end{array}$ & 37.8 & $\mathbf{5 9 . 9}$ & $\mathbf{5 9 . 9 5}$ \\
\hline Top 1 Res $\mu \mathrm{It}$ & $\mathbf{6 0 . 0 1}$ & $\mathbf{6 1 . 9}$ & $\mathbf{6 1 . 9}$ \\
\hline \multicolumn{2}{|c|}{ Adapted Lesk [3] } & 34.05 & 34.05 & 37.8 \\
\hline \multicolumn{2}{|c|}{ First Sense [8] } & 68.8 & 68.8 & 34.05 \\
\hline
\end{tabular}


2. Another research paper was mentioned by Bridget T. McInnes, and Mark Stevenson "Determining the difficulty of Word Sense Disambiguation" 2013 [2].

The problem: Automatic processing of biomedical documents is made difficult by the fact that many of the terms they contain are ambiguous.

Solution: Suggested both unsupervised and supervised methods to estimate the WSD performance on an ambiguous terms of biomedical including abbreviations, phrases and words.

Data Set: MSH-WSD dataset The National Library of Medicine's MSH Word Sense Disambiguation (MSH-WSD) dataset contains 203 ambiguous terms and abbreviations from the 2010 MEDLINE baseline.

Result: Results show that the supervised methods are good predictors of WSD difficulty in some cases, but that their results are not consistent across different data sets. These methods also require labelled training data, limiting their usefulness. The unsupervised approaches do not have this limitation and can be applied to a wider range of ambiguities.

Table 2: Result of WSD accuracy using unsupervised and supervised approaches.

\begin{tabular}{llrr}
\hline \multirow{2}{*}{ Dataset } & Measure & WSD & \\
\cline { 3 - 4 } & & Unsupervised & Supervised \\
\hline \multirow{2}{*}{ NLM-WSD } & \# Senses & $-\mathbf{0 . 3 0}$ & -0.17 \\
& MFS & 0.15 & 0.89 \\
& Entropy & -0.17 & $-\mathbf{0 . 8 8}$ \\
Abbr & \# Senses & $-\mathbf{0 . 4 6}$ & -0.14 \\
& MFS & 0.18 & $-\mathbf{0 . 5 7}$ \\
& Entropy & -0.33 & 0.32 \\
MSH-WSD & \# Senses & -0.05 & -0.06 \\
& MFS & $\mathbf{0 . 1 1}$ & 0.14 \\
& Entropy & $\mathbf{0 . 1 1}$ & $\mathbf{0 . 1 7}$ \\
Combined & \# Senses & $-\mathbf{0 . 1 1}$ & $-\mathbf{0 . 1 1}$ \\
& MFS & -0.05 & 0.03 \\
& Entropy & 0.03 & -0.09 \\
\hline
\end{tabular}


3. Another research work was mentioned by Rada Mihalcea "Using Wikipedia for Automatic Word Sense Disambiguation” 2007, [3].

The problem: Building accurate WSD in automatic way using Wikipedia as a basis of sense annotations.

Solution: proposed a supervised method, where the occurrence of each sense tagged of a specific word is converted into a feature parameter which is then used in an automatic learning process.

Data Set: Nouns of Wikipedia sense corpus and SENSEVAL dataset.

Result: The supervised method relies wholly on monolingual data, which can avoid the possible constraints forced by approaches that need parallel texts, which may be difficult to find.

Table 3: Average number of examples and senses using SENSEVAL and WIKIPEDIA datasets.

\begin{tabular}{lccccc}
\hline & & & \multicolumn{2}{c}{ baselines } & word sense \\
\cline { 4 - 5 } Dataset & \#s & \#ex & MFS & LeskC & disambig. \\
\hline SENSEVAL & 4.60 & 226 & $51.53 \%$ & $58.33 \%$ & $68.13 \%$ \\
WIKIPEDIA & 3.31 & 316 & $72.58 \%$ & $78.02 \%$ & $84.65 \%$ \\
\hline
\end{tabular}


4. Another research paper was studied by Anagha Kulkarni, "Word Sense Disambiguation for Vocabulary Learning”, 2006 [4].

The problem: Improvement of English vocabulary by performing sense disambiguation for homonyms to help ESL students in vocabulary learning.

Solution: Both supervised and unsupervised methods are used in vocabulary learning to find word sense disambiguation. Learning of word meaning pairs was done instead of words.

Data set: 30 homonyms of ESL dictionary.

Result: the result indicates that unsupervised methods were less accurate than supervised approaches. A user study reveals that students equipped with WSD-enabled vocabulary tutor perform significantly better than students using vocabulary tutor without the WSD capabilities.

Table 4: Result of 30 homonyms using supervised classification models.

\begin{tabular}{|c|c|c|c|c|c|c|c|}
\hline & Homonym & $\begin{array}{c}\text { Number } \\
\text { of } \\
\text { senses }\end{array}$ & $\begin{array}{c}\text { Baseline } \\
\text { Accuracy } \\
(\%)\end{array}$ & $\begin{array}{c}\text { Best } \\
\text { Accuracy } \\
(\%)\end{array}$ & $\begin{array}{l}\text { Cohen's } \\
\text { Kappa }\end{array}$ & $\begin{array}{c}\text { Classification } \\
\text { Algorithm }\end{array}$ & $\begin{array}{l}\text { Window } \\
\text { Size (w) }\end{array}$ \\
\hline 1 & panel & 2 & 84.92 & 99.82 & 0.993 & MNB & 25 \\
\hline 2 & transmission & 2 & 51.91 & 99.15 & 0.983 & MNB & 70 \\
\hline 3 & procedure & 2 & 82.04 & 99.40 & 0.980 & MNB & 85 \\
\hline 4 & foundation & 3 & 79.17 & 98.81 & 0.965 & MNB & 85 \\
\hline 5 & principal & 2 & 64.39 & 98.05 & 0.957 & SVM & 40 \\
\hline 6 & bond & 2 & 81.78 & 98.67 & 0.956 & SVM & 70 \\
\hline 7 & aid & 2 & 59.76 & 97.71 & 0.952 & SVM & 85 \\
\hline 8 & tape & 2 & 75.16 & 98.14 & 0.951 & MNB & 40 \\
\hline 9 & monitor & 2 & 84.36 & 98.58 & 0.947 & $\mathrm{MNB}$ & 85 \\
\hline 10 & code & 2 & 66.18 & 97.10 & 0.936 & MNB & 85 \\
\hline 11 & volume & 3 & 51.00 & 96.00 & 0.934 & MNB & 85 \\
\hline 12 & suspend & 2 & 81.48 & 97.53 & 0.919 & MNB & 40 \\
\hline 13 & contract & 3 & 83.67 & 97.73 & 0.919 & MNB & 40 \\
\hline 14 & qualify & 3 & 79.81 & 97.12 & 0.909 & $\mathrm{MNB}$ & 70 \\
\hline 15 & major & 3 & 90.24 & 98.32 & 0.904 & MNB & 40 \\
\hline 16 & conceive & 2 & 80.92 & 96.95 & 0.898 & SVM & 70 \\
\hline 17 & pose & 3 & 58.26 & 94.78 & 0.893 & $\mathrm{MNB}$ & 25 \\
\hline 18 & trigger & 2 & 59.40 & 94.33 & 0.883 & SVM & 25 \\
\hline 19 & brief & 3 & 75.81 & 95.70 & 0.883 & SVM & 10 \\
\hline 20 & parallel & 2 & 53.70 & 94.14 & 0.882 & MNB & 85 \\
\hline 21 & supplement & 2 & 73.18 & 95.45 & 0.882 & MNB & 70 \\
\hline 22 & channel & 2 & 53.25 & 93.49 & 0.869 & MNB & 10 \\
\hline 23 & depress & 2 & 60.66 & 93.44 & 0.862 & MNB & 40 \\
\hline 24 & manual & 2 & 68.80 & 93.59 & 0.850 & SVM & 10 \\
\hline 25 & factor & 2 & 91.24 & 97.72 & 0.848 & MNB & 85 \\
\hline 26 & shift & 3 & 70.71 & 92.55 & 0.837 & MNB & 70 \\
\hline 27 & function & 2 & 90.84 & 97.01 & 0.830 & MNB & 55 \\
\hline 28 & issue & 3 & 80.90 & 92.96 & 0.767 & MNB & 85 \\
\hline 29 & complex & 3 & 58.51 & 86.86 & 0.735 & MNB & 70 \\
\hline 30 & appreciate & 2 & 68.63 & 86.27 & 0.690 & SVM & 40 \\
\hline
\end{tabular}


5. Although, a research paper was mentioned in 2014 by Arindam Roy "Knowledge based approaches to Nepali Word Sense Disambiguation", [7]. They use three types of knowledge base approaches to perform WSD which are semantic graph based, conceptual distance, and overlap based. They use only noun and adjectives of Napali WordNet as data set. The result shows that in overlap based the accuracy were $54 \%$ and $42 \%$ approximately. While the mixture of semantic graph based and conceptual distance resulted to be more accurate than overlap based approach.

6. Another research paper was studied in 2008 by Shrestha [8], they used Lesk algorithm for Nepali WSD. The authors changed the Lesk algorithm in way that just the words in the sentence without their gloss, hypernym, examples and synset are chosen as context words. They use the Nepali WordNet as dataset which resulted this method is more accurate than other approaches.

7. Furthermore, a research was done in 2014 by Dhungana [5], they use adapted Lesk Method to disambiguate the Nepali words. They use 348 words with the test data containing 201 sentences of Nepali language which resulted that this algorithm more accurate and increased by $16.41 \%$ which is better than the accuracy of the approach proposed by Shrestha [8].

8. One more work was mentioned in 2014 by Richard Laishram Singh "A Decision Tree Based WSD System in Manipuri Language" [6]. Proposed contextual and positional features for WSD using a WordNet of Manipuri language as a dataset. 


\section{DISCUSSION}

Three approaches most widely used in word sense disambiguation which are knowledge based approach, supervised approach and unsupervised approach, here will discuss the advantages and disadvantage of each approach.

Table 5: Advantages and disadvantages of WSD approaches

\begin{tabular}{|l|l|l|}
\hline Approach & Advantage & Disadvantage \\
\hline $\begin{array}{l}\text { Knowledge- } \\
\text { Based }\end{array}$ & $\begin{array}{l}\text { These algorithms give higher } \\
\text { Precision. }\end{array}$ & $\begin{array}{l}\text { These algorithms are overlap based, } \\
\text { so they suffer from overlap sparsity } \\
\text { and performance depends on } \\
\text { dictionary definitions. }\end{array}$ \\
\hline Supervised & $\begin{array}{l}\text { This type of algorithms are } \\
\text { better than the two } \\
\text { approaches } \\
\text { implementation perspective. }\end{array}$ & $\begin{array}{l}\text { These algorithms don't give } \\
\text { satisfactory result for resource } \\
\text { scarce languages. }\end{array}$ \\
\hline Unsupervised & $\begin{array}{l}\text { There is no need of any sense } \\
\text { inventory and sense } \\
\text { annotated corpora in these } \\
\text { approaches. }\end{array}$ & $\begin{array}{l}\text { These algorithms are difficult to } \\
\text { implement and performance is } \\
\text { always inferior to that of other two } \\
\text { approaches. }\end{array}$ \\
\hline
\end{tabular}

\section{CONCLUSION}

In this study, we have reviewed the task of word sense disambiguation, WSD is the toughest open problems of NLP. Some of WSD challenges has been discussed, many problems occurs in WSD because it depends on knowledge drawn from different resources. WSD also deals with complexities of languages. Knowledge based, supervised approach, unsupervised approach and semi supervised approaches are used in WSD. Supervised approach performs well as a compared to all other approach because training data totally depends on specific domain. 


\section{References:}

[1] Sachdeva, Pradeep, Surbhi Verma, and Sandeep Kumar Singh. "An improved approach to word sense disambiguation", 2014 IEEE International Symposium on Signal Processing and Information Technology (ISSPIT), 2014.

[2] Bridget T. McInnes, and Mark Stevenson, "Determining the difficulty of Word Sense Disambiguation" Journal of Biomedical Informatics 47 (2014) 83-90

[3] Rada Mihalcea "Using Wikipedia for Automatic Word Sense Disambiguation" Association for Computational Linguistics, Proceedings of NAACL HLT 2007, pages 196-203, Rochester, NY, April 2007.

[4] Anagha Kulkarni, Michael Heilman, Maxine Eskenazi and Jamie Callan, 2006, "Word Sense Disambiguation for Vocabulary Learning", Proceedings of the Ninth International Conference on Spoken Language Processing, 2006.

[5] U. R. Dhungana and S. Shakya, "Word sense disambiguation in nepali language," in The Fourth International Conference on Digital Information and Communication Technology and Its Application (DICTAP2014), Bangkok, Thailand, 2014, pp. 46-50.

[6] Richard Laishram Singh, Krishnendu Ghosh, Kishorjit Nongmeikapam and Sivaji Bandyopadhyay, "A Decision Tree Based Word Sense Disambiguation System in Manipuri Language", Proceedings of International Conference of Natural Language Processing and Cognitive Computing, 2014.

[7] Arindam Roy, Sunita Sarkar, Bipul Syam Purkayastha, "Knowledge based approaches to Nepali Word Sense Disambiguation", Proceedings of International Conference of Natural Language Processing and Cognitive Computing, India, 2014.

[8] N. Shrestha, A. V. H. Patrick, and S. K. Bista, "Resources for nepali word sense disambiguation," in IEEE International conference on Natural Language Processing and Knowledge Engineering (IEEE NLP-KE'08), Beijing, China, 2008.

[9] N. Omar and Q. Al-Tashi, "Arabic nested noun compound extraction based on linguistic features and

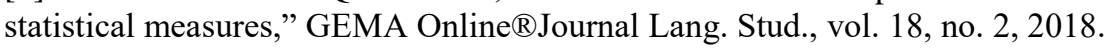

[10] Q. Al-Tashi, S. J. A. Kadir, H. M. Rais, S. Mirjalili, and H. Alhussian, "Binary Optimization Using Hybrid Grey Wolf Optimization for Feature Selection,” IEEE Access, vol. 7, pp. 39496-39508, 2019.

[11] Q. Al-Tashi, H. Rais, and S. Jadid, "Feature Selection Method Based on Grey Wolf Optimization for Coronary Artery Disease Classification," in International Conference of Reliable Information and Communication Technology, 2018, pp. 257-266.

[12] Q. Al-Tashi, H. Rais, and S. J. Abdulkadir, "Hybrid Swarm Intelligence Algorithms with Ensemble Machine Learning for Medical Diagnosis," in 2018 4th International Conference on Computer and Information Sciences (ICCOINS), 2018, pp. 1-6.

[13] Fang, J., Lin, D., Schulz, S.C., Xu, Z., Calhoun, V.D., and Wang, Y.-P.J.B. (2016). Joint sparse canonical correlation analysis for detecting differential imaging genetics modules. 32(22), 3480-3488.

[14] F. Al-shargie, T. Tang, N. Badruddin, and M. Kiguchi, "Mental Stress Quantification Using EEG Signals," in International Conference for Innovation in Biomedical Engineering and Life Sciences, 2016, pp. 1519.

[15] Al-Shargie, Fares. "Early Detection of Mental Stress Using Advanced Neuroimaging and Artificial Intelligence." arXiv preprint arXiv:1903.08511 (2019).

[16] Al-Shargie, Fares. "Fusion of fNIRS and EEG Signals: Mental Stress Study." (2019).

[17] Al-Shargie, Fares. "Multilevel Assessment of Mental Stress using SVM with ECOC: An EEG Approach." (2019).

[18] Al-Shargie, Fares. "Quantification of Mental Stress using fNIRS Signals." (2019).

[19] Al-Shargie, Fares, Tong Boon Tang, Nasreen Badruddin, and Masashi Kiguchi. "Towards multilevel mental stress assessment using SVM with ECOC: an EEG approach." Medical \& biological engineering \& computing 56, no. 1 (2018): 125-136.

[20] Al-Shargie, Fares, Tong Boon Tang, and Masashi Kiguchi. "Assessment of mental stress effects on prefrontal cortical activities using canonical correlation analysis: an fNIRS-EEG study." Biomedical optics express 8, no. 5 (2017): 2583-2598. 
[21] Al-Shargie, Fares, Tong Boon Tang, and Masashi Kiguchi. "Stress assessment based on decision fusion of EEG and fNIRS signals." IEEE Access 5 (2017): 19889-19896.

[22] Al-Shargie, Fares, Masashi Kiguchi, Nasreen Badruddin, Sarat C. Dass, Ahmad Fadzil Mohammad Hani, and Tong Boon Tang. "Mental stress assessment using simultaneous measurement of EEG and fNIRS." Biomedical optics express 7, no. 10 (2016): 3882-3898.

[23] Al-shargie, Fares, Tong Boon Tang, Nasreen Badruddin, Sarat C. Dass, and Masashi Kiguchi. "Mental stress assessment based on feature level fusion of fNIRS and EEG signals." In 2016 6th International Conference on Intelligent and Advanced Systems (ICIAS), pp. 1-5. IEEE, 2016.

[24] Al-shargie, Fares, Tong Boon Tang, and Masashi Kiguchi. "Mental stress grading based on fNIRS signals." In 2016 38th Annual International Conference of the IEEE Engineering in Medicine and Biology Society (EMBC), pp. 5140-5143. IEEE, 2016.

[25] Al-Shargie, F. M., Tong Boon Tang, Nasreen Badruddin, and Masashi Kiguchi. "Mental stress quantification using EEG signals." In International Conference for Innovation in Biomedical Engineering and Life Sciences, pp. 15-19. Springer, Singapore, 2015.

[26] Al-shargie, Fares, Tong Boon Tang, Nasreen Badruddin, and Masashi Kiguchi. "Simultaneous measurement of EEG-fNIRS in classifying and localizing brain activation to mental stress." In 2015 IEEE International Conference on Signal and Image Processing Applications (ICSIPA), pp. 282-286. IEEE, 2015.

[27] Mohamed, Eltaf Abdalsalam, Mohd Zuki Yusoff, Ibrahim Khalil Adam, Elnazeer Ali Hamid, Fares AlShargie, and Muhammad Muzammel. "Enhancing EEG Signals in Brain Computer Interface Using Intrinsic TimeScale Decomposition." In Journal of Physics: Conference Series, vol. 1123, no. 1, p. 012004. IOP Publishing, 2018.

[28] Al-Shargie, Fares. "Assessment of Mental Stress Using EEG and fNIRS Features." (2019). 